\title{
Statistical Abstraction and Model-Checking of Large Heterogeneous Systems ${ }^{\star}$
}

\author{
Ananda Basu ${ }^{1}$, Saddek Bensalem ${ }^{1}$, Marius Bozga ${ }^{1}$, Benoît Caillaud ${ }^{2}$, \\ Benoît Delahaye $^{3}$, and Axel Legay ${ }^{2}$ \\ 1 Verimag Laboratory, Université Joseph Fourier Grenoble, CNRS \\ ${ }^{2}$ INRIA/IRISA, Rennes, France \\ 3 Université de Rennes 1/IRISA, Rennes, France
}

\begin{abstract}
We propose a new simulation-based technique for verifying applications running within a large heterogeneous system. Our technique starts by performing simulations of the system in order to learn the context in which the application is used. Then, it creates a stochastic abstraction for the application, which takes the context information into account. This smaller model can be verified using efficient techniques such as statistical model checking. We have applied our technique to an industrial case study: the cabin communication system of an airplane. We use the BIP toolset to model and simulate the system. We have conducted experiments to verify the clock synchronization protocol i.e., the application used to synchronize the clocks of all computing devices within the system.
\end{abstract}

\section{Introduction}

Systems integrating multiple heterogeneous distributed applications communicating over a shared network are typical in various sensitive domains such as aeronautic or automotive embedded systems. Verifying the correctness of a particular application inside such a system is known to be a challenging task, which is often beyond the scope of existing exhaustive validation techniques. The main difficulty comes from network communication which makes all applications interfering and therefore forces exploration of the full state-space of the system.

Statistical Model Checking 813 15] has recently been proposed as an alternative to avoid an exhaustive exploration of the state-space of the model. The core idea of the approach is to conduct some simulations of the system and then use statistical results in order to decide whether the system satisfies the property or not. Statistical model checking techniques can also be used to estimate the probability that a system satisfies a given property [8]. Of course, in contrast with an exhaustive approach, a simulation-based solution does not guarantee a correct result. However, it is possible to bound the probability of making an error. Simulation-based methods are known to be far less memory and time intensive than exhaustive ones, and are sometimes the only option [1610. Statistical model checking is widely accepted in various research areas such as systems

\footnotetext{
^ This work has been supported by the Combest EU project.
} 
biology [6]11] or software engineering, in particular for industrial applications. There are several reasons for this success. First, it is very simple to implement, understand and use. Second, it does not require extra modeling or specification effort, but simply an operational model of the system, that can be simulated and checked against state-based properties. Third, it allows model-checking of properties [5] that cannot be expressed in classical temporal logics. Nevertheless, statistical-model checking still suffers from the system's complexity. In particular, for the case of heterogeneous systems, the number of components and their interactions are limiting factors on the number and length of simulations that can be conducted and hence on the accuracy of the statistical estimates.

We propose to exploit the structure of the system in order to increase the efficiency of the verification process. The idea is conceptually simple: instead of performing an analysis of the entire system, we propose to analyze each application separately, but under some particular context/execution environment. This context is a stochastic abstraction that represents the interactions with other applications running within the system and sharing the computation and communication resources. We propose to build such a context automatically by simulating the system and learning the probability distributions of key characteristics impacting the functionality of the given application.

The overall contribution of this paper is an application of the above method on an industrial case study, the heterogeneous communication system (HCS for short) deployed for cabin communication in a civil airplane. HCS is a heterogeneous system providing entertainment services (e.g., audio/video on passengers demand) as well as administrative services (e.g., cabin illumination, control, audio announcements), which are implemented as distributed applications running in parallel, across various devices within the plane and communicating through a common Ethernet-based network. The HCS system has to guarantee stringent requirements, such as reliable data transmission, fault tolerance, timing and synchronization constraints. An important requirement, which will be studied in this paper, is the accuracy of clock synchronization between different devices. This latter property states that the difference between the clocks of any two devices should be bounded by a small constant, which is provided by the user and depends on his needs. Hence, one must be capable of computing the smallest bound for which synchronization occurs and compare it with the bound expected by the user. Unfortunately, due to the large number of heterogeneous components that constitute the system, deriving such a bound manually from the textual specification is an unfeasible task. In this paper, we propose a formal approach that consists in building a formal model of the HCS, then applying simulation-based algorithms to this model in order to deduce the smallest value of the bound for which synchronization occurs. We start with a fixed value of the bound and check whether synchronization occurs. If yes, then we make sure that this is the best one. If no, we restart the experiment with a new value.

At the top of our approach, there should be a tool that is capable of modeling heterogeneous systems as well as simulating their executions and the interactions between components. In this paper, we propose to use the BIP toolset [2] 
for doing so. BIP (Behaviour-Interaction-Priority) supports a methodology for building systems from atomic components encapsulating behavior, that communicate through interactions, and coordinate through priorities. BIP also offers a powerful engine to simulate the system and can thus be combined with a statistical model checking algorithm in order to verify properties. Our first contribution is to study all the requirements for the HCS to work properly and then derive a model in BIP. Our second contribution is to study the accuracy of clock synchronization between several devices of the HCS. In HCS the clock synchronization is ensured by the Precision Time Protocol (PTP for short) 1, and the challenge is to guarantee that PTP maintains the difference between a master clock (running on a designated server within the system) and all the slave clocks (running on other devices) under some bound. Since this bound cannot be pre-computed, we have to verify the system for various values of the bound until we find a suitable one. Unfortunately, the full system is too big to be analyzed with classical exhaustive verification techniques. A solution could be to remove all the information that is not related to the devices under consideration. This is in fact not correct as the behavior of the PTP protocol is influenced by the other applications running in parallel within the heterogeneous system. Our solution to this state-space explosion problem is in two steps (1) we will build a stochastic abstraction for a part of the PTP application between the server and a given device; the stochastic part will be used to model the general context in which PTP is used, (2) we will apply statistical model checking on the resulting model.

Thanks to this approach, we have been able to derive precise bounds that guarantee proper synchronization for all the devices of the system. We also computed the probability of satisfying the property for smaller values of the bound, i.e., bounds that do not satisfy the synchronization property with probability 1 . Being able to provide such information is of clear importance, especially when the best bound is too high with respect to the user's requirements. We have observed that the values we obtained strongly depend on the position of the device in the network. We also estimated the average and worst proportion of failures per simulation for bounds that are smaller than the one that guarantees synchronization. Checking this latter property has been made easy because BIP allows us to reason on one execution at a time. Finally, we have also considered the influence of clock drift on the synchronisation results. The experiments highlight the generality of our technique, which could be applied to other versions of the HCS as well as to other heterogeneous applications.

Due to space limitations, several constructions and algorithms are given in a technical report [3].

\section{An Overview of Statistical Model Checking}

Consider a stochastic system $\mathcal{S}$ and a property $\phi$. Statistical model checking refers to a series of simulation-based techniques that can be used to answer two questions: (1) Qualitative: Is the probability that $\mathcal{S}$ satisfies $\phi$ greater or equal 
to a certain threshold? and (2) Quantitative: What is the probability that $\mathcal{S}$ satisfies $\phi$ ? Contrary to numerical approaches, the answer is given up to some correctness precision. In the rest of the section, we overview several statistical model checking techniques. Let $B_{i}$ be a discrete random variable with a Bernoulli distribution of parameter $p$. Such a variable can only take 2 values 0 and 1 with $\operatorname{Pr}\left[B_{i}=1\right]=p$ and $\operatorname{Pr}\left[B_{i}=0\right]=1-p$. In our context, each variable $B_{i}$ is associated with one simulation of the system. The outcome for $B_{i}$, denoted $b_{i}$, is 1 if the simulation satisfies $\phi$ and 0 otherwise.

\subsection{Qualitative Answer Using Statistical Model Checking}

The main approaches [1513] proposed to answer the qualitative question are based on hypothesis testing. Let $p=\operatorname{Pr}(\phi)$, to determine whether $p \geq \theta$, we can test $H: p \geq \theta$ against $K: p<\theta$. A test-based solution does not guarantee a correct result but it is possible to bound the probability of making an error. The strength $(\alpha, \beta)$ of a test is determined by two parameters, $\alpha$ and $\beta$, such that the probability of accepting $K$ (respectively, $H$ ) when $H$ (respectively, $K$ ) holds, called a Type-I error (respectively, a Type-II error ) is less or equal to $\alpha$ (respectively, $\beta$ ). A test has ideal performance if the probability of the Type-I error (respectively, Type-II error) is exactly $\alpha$ (respectively, $\beta$ ). However, these requirements make it impossible to ensure a low probability for both types of errors simultaneously (see [15] for details). A solution is to use an indifference region $\left[p_{1}, p_{0}\right]$ (with $\theta$ in $\left[p_{1}, p_{0}\right]$ ) and to test $H_{0}: p \geq p_{0}$ against $H_{1}: p \leq p_{1}$. We now sketch two hypothesis testing algorithms.

Single Sampling Plan. To test $H_{0}$ against $H_{1}$, we specify a constant $c$. If $\sum_{i=1}^{n} b_{i}$ is larger than $c$, then $H_{0}$ is accepted, else $H_{1}$ is accepted. The difficult part in this approach is to find values for the pair $(n, c)$, called a single sampling plan (SSP in short), such that the two error bounds $\alpha$ and $\beta$ are respected. In practice, one tries to work with the smallest value of $n$ possible so as to minimize the number of simulations performed. Clearly, this number has to be greater if $\alpha$ and $\beta$ are smaller but also if the size of the indifference region is smaller. This results in an optimization problem, which generally does not have a closed-form solution except for a few special cases [15]. In his thesis [15], Younes proposes a binary search based algorithm that, given $p_{0}, p_{1}, \alpha, \beta$, computes an approximation of the minimal value for $c$ and $n$.

Sequential probability ratio test. The sample size for a single sampling plan is fixed in advance and independent of the observations that are made. However, taking those observations into account can increase the performance of the test. As an example, if we use a single plan $(n, c)$ and the $m>c$ first simulations satisfy the property, then we could (depending on the error bounds) accept $H_{0}$ without observing the $n-m$ other simulations. To overcome this problem, one can use the sequential probability ratio test (SPRT in short) proposed by Wald 14. The approach is briefly described below. 
In SPRT, one has to choose two values $A$ and $B(A>B)$ that ensure that the strength of the test is respected. Let $m$ be the number of observations that have been made so far. The test is based on the following quotient:

$$
\frac{p_{1 m}}{p_{0 m}}=\prod_{i=1}^{m} \frac{\operatorname{Pr}\left(B_{i}=b_{i} \mid p=p_{1}\right)}{\operatorname{Pr}\left(B_{i}=b_{i} \mid p=p_{0}\right)}=\frac{p_{1}^{d_{m}}\left(1-p_{1}\right)^{m-d_{m}}}{p_{0}^{d_{m}}\left(1-p_{0}\right)^{m-d_{m}}},
$$

where $d_{m}=\sum_{i=1}^{m} b_{i}$. The idea behind the test is to accept $H_{0}$ if $\frac{p_{1 m}}{p_{0 m}} \geq A$, and $H_{1}$ if $\frac{p_{1 m}}{p_{0 m}} \leq B$. The SPRT algorithm computes $\frac{p_{1 m}}{p_{0 m}}$ for successive values of $m$ until either $H_{0}$ or $H_{1}$ is satisfied; the algorithm terminates with probability 1 [14. This has the advantage of minimizing the number of simulations. In his thesis [15, Younes proposed a logarithmic based algorithm SPRT that given $p_{0}, p_{1}, \alpha$ and $\beta$ implements the sequential ratio testing procedure.

\subsection{Quantitative Answer Using Statistical Model Checking}

In 812 Peyronnet et al. propose an estimation procedure to compute the probability $p$ for $\mathcal{S}$ to satisfy $\phi$. Given a precision $\delta$, Peyronnet's procedure, which we call PESTIMATION, computes a value for $p^{\prime}$ such that $\left|p^{\prime}-p\right| \leq \delta$ with confidence $1-\alpha$. The procedure is based on the Chernoff-Hoeffding bound 9 . Let $B_{1} \ldots B_{m}$ be $m$ discrete random variables with a Bernoulli distribution of parameter $p$ associated with $m$ simulations of the system. Recall that the outcome for each of the $B_{i}$, denoted $b_{i}$, is 1 if the simulation satisfies $\phi$ and 0 otherwise. Let $p^{\prime}=\left(\sum_{i=1}^{m} b_{i}\right) / m$, then Chernoff-Hoeffding bound [9] gives $\operatorname{Pr}\left(\left|p^{\prime}-p\right|>\delta\right)<2 e^{-\frac{m \delta^{2}}{4}}$. As a consequence, if we take $m \geq \frac{4}{\delta^{2}} \log \left(\frac{2}{\alpha}\right)$, then $\operatorname{Pr}\left(\left|p^{\prime}-p\right| \leq \delta\right) \geq 1-\alpha$. Observe that if the value $p^{\prime}$ returned by PESTIMATION is such that $p^{\prime} \geq \theta-\delta$, then $\mathcal{S} \models P r_{\geq \theta}$ with confidence $1-\alpha$.

\subsection{Playing with Statistical Model Checking Algorithms}

The efficiency of the above algorithms is characterized by the number of simulations needed to obtain an answer. This number may change from executions to executions and can only be estimated (see [15] for an explanation). However, some generalities are known. For the qualitative case, it is known that, except for some situations, SPRT is always faster than SSP. When $\theta=1$ (resp. $\theta=0$ ) SPRT degenerates to SSP; this is not problematic since SSP is known to be optimal for such values. PESTIMATION can also be used to solve the qualitative problem, but it is always slower than SSP [15]. If $\theta$ is unknown, then a good strategy is to estimate it using PESTIMATION with a low confidence and then validate the result with SPRT and a strong confidence.

\section{Validation Method and the BIP Toolset}

Consider a system consisting of a set of distributed applications running on several computers and exchanging messages on a shared network infrastructure. 
Assume also that network communication is subject to given bandwidth restrictions as well as to routing and scheduling policies applied on network elements. Our method attempts to reduce the complexity of validation of a particular application of such system by decoupling the timing analysis of the network and functional analysis of each application.

We start by constructing a model of the whole system. This model must be executable, i.e., it should be possible to obtain execution traces, annotated with timing information. For a chosen application, we then learn the probability distribution laws of its message delays by simulating the entire system. The method then constructs a reduced stochastic model by combining the application model where the delays are defined according to the laws identified at the previous step. Finally, the method applies statistical model-checking on the resulting stochastic model.

Our models are specified within the BIP framework [2]. BIP is a componentbased framework for construction, implementation and analysis of systems composed of heterogeneous components. In particular, BIP fulfills all the requirements of the method suggested above, that are, models constructed in BIP are operational and can be thoroughly simulated. BIP models can easily integrate timing constraints, which are represented with discrete clocks. Probabilistic behaviour can also be added by using external $\mathrm{C}$ functions.

The BIP framework is implemented within the BIP toolset 4], which includes a rich set of tools for modeling, execution, analysis (both static and on-the-fly) and static transformations of BIP models. It provides a dedicated programming language for describing BIP models. The front-end tools allow editing and parsing of BIP programs, and generating an intermediate model, followed by code generation (in $\mathrm{C}$ ) for execution and analysis on a dedicated middleware platform. The platform also offers connections to external analysis tools. A more complete description of BIP can be found in [3].

\section{Case Study: Heterogeneous Communication System}

The case study concerns a distributed heterogeneous communication system (HCS) providing an all electronic communication infrastructure to be deployed, typically for cabin communication in airplanes or for building automation. The HCS system contains various devices such as sensors (video camera, smoke detector, temperature, pressure, etc.) and actuators (loudspeakers, light switches, temperature control, signs, etc.) connected through a wired communication network to a common server. The server runs a set of services to monitor the sensors and to control the actuators. The devices are connected to the server using network access controllers (NAC) as shown for an example architecture in Figure 1 An extended star-like HCS architecture with several daisy chains of NACs and devices is presented in [3].

The system-wide HCS architecture is highly heterogeneous. It includes hardware components and software applications ensuring functions with different characteristics and degree of criticality e.g., audio streaming, device clock synchronisation, sensor monitoring, video surveillance. It also integrates different 


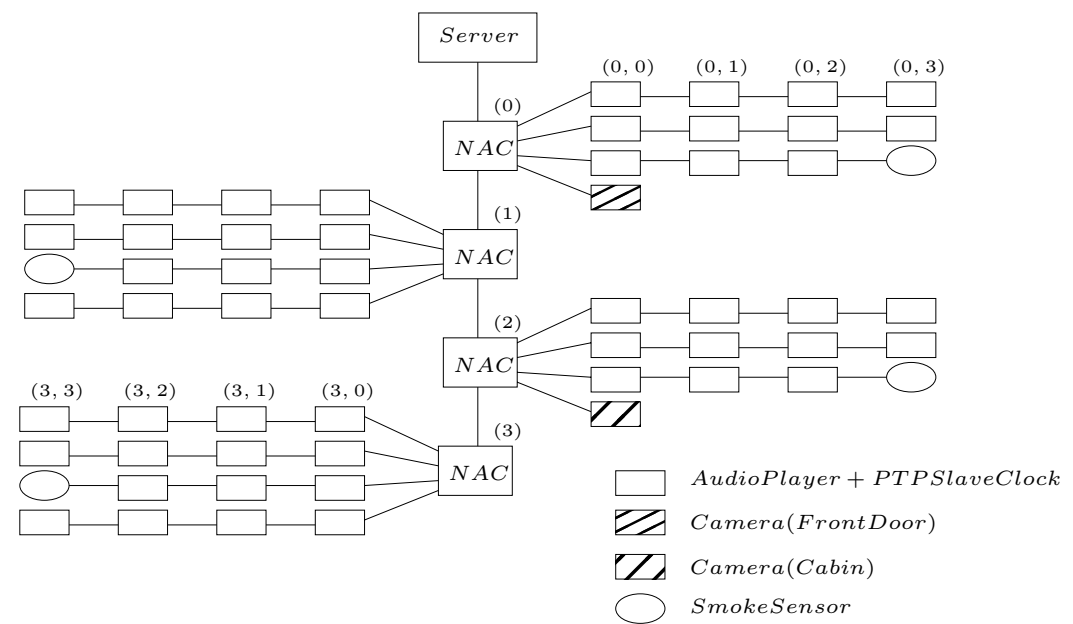

Fig. 1. HCS Example Model

communication and management protocols between components. The HCS system has to guarantee stringent requirements, such as reliable data transmission, fault tolerance, timings and synchronization constraints. For example, the latency for delivering alarm signals from sensors, or for playing audio announcements should be smaller than certain predefined thresholds. Or, the accuracy of clock synchronization between different devices, should be guaranteed under the given physical implementation of the system.

The HCS case study poses challenges that require component-based design techniques, since it involves heterogeneous components and communication mechanisms, e.g. streaming based on the data-flow paradigm as well as event driven computation and interaction. Its modeling needs combination of executable and analytic models especially for performance evaluation and analysis of non-functional properties.

We have modeled an instance of the HCS system in BIP. As shown in Figure 1, the system consists of one Server connected to a daisy chain of four NACs, addressed $0 \cdots 3$, and several devices. Devices are connected in daisy chains with the NACs, the length of each chain being limited to four in our example. For simplicity, devices are addressed $(i, j)$, where $i$ is the address of the NAC and $j$ is the address of the device. The model contains three types of devices, namely $A u$ dio Player, Video Camera and Smoke Sensor. The devices connected to NAC(0) and NAC(2) have similar topology. The first two daisy-chains consist of only Audio Player devices. The third daisy-chain ends with a Smoke Sensor, and the fourth daisy-chain consists of just one Video Camera. The devices connected to $\mathrm{NAC}(1)$ and $\mathrm{NAC}(3)$ have exactly the same topology, consisting of several Audio Player and one Smoke Sensor devices.

The system depicted in Figure 1 contains 58 devices in total. The BIP model contains 297 atomic components, 245 clocks, and its state-space is of order $2^{3000}$. 
The size of the BIP code for describing the system is 2468 lines, which is translated to 9018 lines in C. A description of the key components is given in [3].

\section{Experiments on the HCS}

One of the core applications of the HCS case study is the PTP protocol, which allows the synchronization of the clocks of the various devices with the one of the server. It is important that this synchronization occurs properly, i.e., the difference between the clock of the server and the one of any device is bounded by a small constant. Studying this problem is the subject of this section. Since the BIP model for the HCS is extremely large (number of components, size of the state space, ...), there is no hope to analyse it with an exhaustive verification technique. Here, we propose to apply our stochastic abstraction. Given a specific device, we will proceed in two steps. First, we will conduct simulations on the entire system in order to learn the probability distribution on the communication delays between this device and the server. Second, we will use this information to build a stochastic abstraction of the application on which we will apply statistical model checking. We start with the stochastic abstraction for the PTP.

\subsection{The Precision Time Protocol IEEE 1588}

The Precision Time Protocol [1] has been defined to synchronize clocks of several computers interconnected over a network. The protocol relies on multicast communication to distribute a reference time from an accurate clock (the master) to all other clocks in the network (the slaves) combined with individual offset correction, for each slave, according to its specific round-trip communication delay to the master. The accuracy of synchronization is negatively impacted by the jitter (i.e., the variation) and the asymmetry of the communication delay between the master and the slaves. Obviously, these delay characteristics are highly dependent on the network architecture as well as on the ongoing network traffic.

We present below the abstract stochastic model of the PTP protocol between a device and the server in the HCS case study. The model consists of two (deterministic) application components respectively, the master and the slave clocks, and two probabilistic components, the media, which are abstraction of the communication network between the master and the slave. The former represent the behaviour of the protocol and are described by extended timed i/o-automata. The latter represent a random transport delay and are simply described by probabilistic distributions. Recap that randomization is used to represent the context, i.e., behaviors of other devices and influence of these behaviors on those of the master and the device under consideration.

The time of the master process is represented by the clock variable $\theta_{m}$. This is considered the reference time and is used to synchronize the time of the slave clock, represented by the clock variable $\theta_{s}$. The synchronization works as follows. Periodically, the master broadcast a sync message and immediately after a followUp message containing the time $t_{1}$ at which the sync message has been sent. 


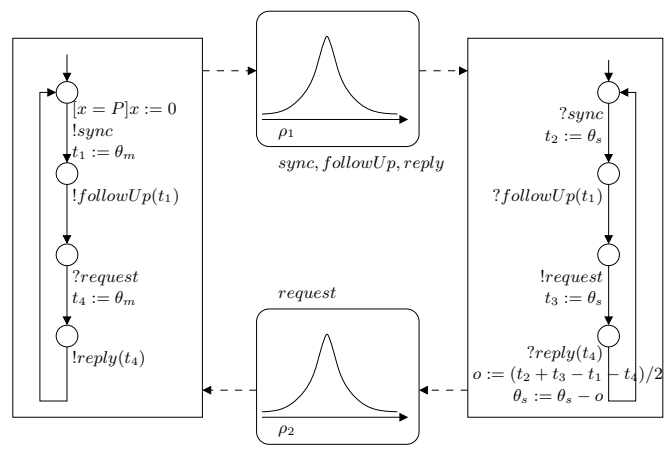

Fig. 2. Abstract stochastic PTP between the server and a device

Time $t_{1}$ is observed on the master clock $\theta_{m}$. The slave records in $t_{2}$ the reception time of the sync message. Then, after the reception of the followUp, it sends a delay request message to the master and records its emission time $t_{3}$. Both $t_{2}$ and $t_{3}$ are observed on the slave clock $\theta_{s}$. The master records on $t_{4}$ the reception time of the request message and sends it back to the slave on the reply message. Again, $t_{4}$ is observed on the master clock $\theta_{m}$. Finally, upon reception of reply, the slave computes the offset between its time and the master time based on $\left(t_{i}\right)_{i=1,4}$ and updates its clock accordingly. In our model, the offset is computed differently in two different situations. In the first situation, which is depicted in Figure 2 the average delays from master to slave and back are supposed to be equal i.e., $\mu\left(\rho_{1}\right)=\mu\left(\rho_{2}\right)$. In the second situation, delays are supposed to be asymmetric, i.e., $\mu\left(\rho_{1}\right) \neq \mu\left(\rho_{2}\right)$. In this case, synchronization is improved by using an extra offset correction which compensate for the difference, more precisely, $o:=\left(t_{2}+t_{3}-t_{1}-t_{4}\right) / 2+\left(\mu\left(\rho_{2}\right)-\mu\left(\rho_{1}\right)\right) / 2$. This offset computation is an extension of the PTP specification and has been considered since it ensures better precision when delays are not symmetric (see Section 5).

Encoding the abstract model of timed i/o-automata given in Figure 2 in BIP is quite straightforward and can be done with the method presented in [2]. The distribution on the delay is implemented as a new $\mathrm{C}$ function in the BIP model. It is worth mentioning that, since the two i/o automata are deterministic, the full system depicted in Figure 2 is purely stochastic.

The accuracy of the synchronization is defined by the absolute value of the difference between the master and slave clocks $\left|\theta_{m}-\theta_{s}\right|$, during the time. Our aim is to check the (safety) property of bounded accuracy $\phi_{\Delta}$, that is, always $\left|\theta_{m}-\theta_{s}\right| \leq \Delta$ for arbitrary fixed non-negative real $\Delta$.

Finally, a simpler version of this protocol has been considered (see [3]) and analyzed. In that study, delay components have been modeled using nondeterministic timed i/o automata as well and represent arbitrary delays bounded in some intervals $[L, U]$. It has been shown that, if the clock drift is negligible, the best accuracy $\Delta^{\star}$ that can be obtained using PTP is respectively $\frac{U-L}{2}$ in the symmetric case, and $\frac{U_{1}+U_{2}-L_{1}-L_{2}}{4}$ in the asymmetric case. That is, the property of bounded accuracy holds trivially iff $\Delta \geq \Delta^{*}$. 


\subsection{Model Simulations}

In this section, we describe our approach to learn the probability distribution over the delays. Consider the server and a given device. In a first step, we run simulations on the system and measure the end-to-end delays of all PTP messages between the selected device and the server. For example, consider the case of delay request messages and assume that we made 33 measures. The result will be a series of delay values and, for each value, the number of times it has been observed. As an example, delay 5 has been observed 3 times, delay 19 has been observed 30 times. The probability distribution is represented with a table of 33 cells. In our case, 3 cells of the table will contains the value 5 and 30 will contain the value 19. The BIP engine will select a value in the table following a uniform probability distribution.

According to our experiments, 2000 delay measurements are enough to obtain an accurate estimation of the probability distribution. However, for confidence reasons, we have conducted 4000 measurements. We have also observed that the value of the distribution clearly depends on the position of the device in the topology (see [3] for an illustration). It is worth mentioning that running one single simulation allowing 4000 measurements of the delay of PTP frames requires running the $\mathrm{PTP}$ protocol with an increased frequency i.e., the default PTP period (2 minutes) being far too big compared with the period for sending audio/video packets (tens of milliseconds). Therefore, we run simulations where PTP is executed once every 2 milliseconds and, we obtain 4000 measurements by simulating approximately 8 seconds of the global system lifetime. Each simulation uses microsecond time granularity and takes around 40 minutes on a Pentium 4 running under a Linux distribution.

\subsection{Experiments on Precision Estimation for PTP}

Three sets of experiments are conducted. The first one is concerned with the bounded accuracy property (see Section 5.1). In the second one, we study average failure per execution for a given bound. Finally, we study the influence of drift on the results.

Property 1: Synchronization. Our objective is to compute the smallest bound $\Delta$ under which synchronization occurs properly for any device. We start with an experiment that shows that the value of the bound depends on the place of the device in the topology. For doing so, we use $\Delta=50 \mu \mathrm{s}$ as a bound and then compute the probability for synchronization to occur properly for all the devices. In the paper, for the sake of presentation, we will only report on a sampled set of devices: $(0,0),(0,3),(1,0),(1,10),(2,0),(2,3),(3,0),(3,3)$, but our global observations extend to any device. We use PESTIMATION with a confidence of 0.1 . The results, which are reported in Figure 3a, show that the place in the topology plays a crucial role. Device $(3,3)$ has the best probability value and Device $(2,0)$ has the worst one. All the results in Figure $3 \mathrm{a}$ have been conducted on the model with asymmetric delays. For the symmetric case, the probability 


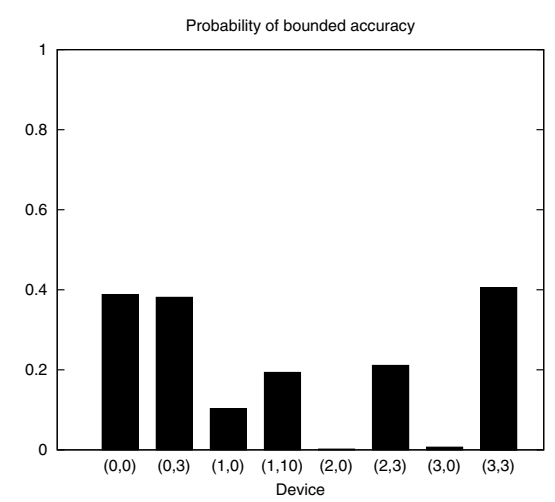

(a) Probability of satisfying bounded accuracy.

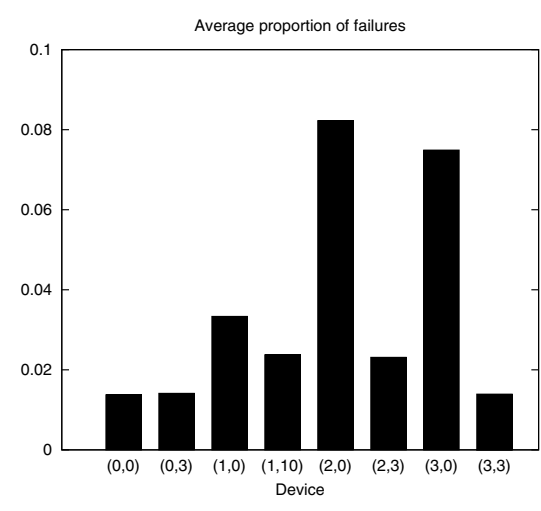

(b) Average proportion of failures.

Fig. 3. Probability of satisfying the bounded accuracy property and average proportion of failures for a bound $\Delta=50 \mu s$ and the asymmetric version of PTP

values are much smaller. As an example, for Device $(0,0)$, it decreases from 0.388 to 0.085 . The above results have been obtained in less than 4 seconds. As a second experiment, we have used SPRT and SSP to validate the probability value found by PESTIMATION with a higher degree of confidence. The results, which are presented in Table 1 for Device $(0,0)$, show that SPRT is faster than SSP and PESTIMATION.

Table 1. Number of simulations / Amount of time required for PESTIMATION, SSP and SPRT

\begin{tabular}{l|c|c|c|c|c|c|}
\multirow{2}{*}{ Precision } & \multicolumn{2}{|c|}{$10^{-1}$} & \multicolumn{2}{c|}{$10^{-2}$} & \multicolumn{2}{c|}{$10^{-3}$} \\
\hline Confidence & $10^{-5}$ & $10^{-10}$ & $10^{-5}$ & $10^{-10}$ & $10^{-5}$ & $10^{-10}$ \\
\hline \hline \multirow{2}{*}{ PESTIMATION } & 4883 & 9488 & 488243 & 948760 & 48824291 & 94875993 \\
& $17 s$ & $34 s$ & $29 m$ & $56 m$ & $>3 h$ & $>3 h$ \\
\hline \multirow{2}{*}{ SSP } & 1604 & 3579 & 161986 & 368633 & 16949867 & 32792577 \\
& $10 s$ & $22 s$ & $13 m$ & $36 m$ & $>3 h$ & $>3 h$ \\
\hline \multirow{2}{*}{ SPRT } & 316 & 1176 & 12211 & 22870 & 148264 & 311368 \\
& $2 s$ & $7 s$ & $53 s$ & $1 m 38 s$ & $11 m$ & $31 m$ \\
\hline
\end{tabular}

Our second step was to estimate the best bound. For doing so, for each device we have repeated the previous experiments for values of $\Delta$ between $10 \mu \mathrm{s}$ and $120 \mu \mathrm{s}$. Figure $4 \mathrm{a}$ gives the results of the probability of satisfying the bounded accuracy property as a function of the bound $\Delta$ for the asymmetric version of PTP. The figure shows that the smallest bound which ensure synchronization for any device is $105 \mu$ s (for Device $(3,0)$ ). However, devices $(0,3)$ and $(3,3)$ already satisfy the property with probability 1 for $\Delta=60 \mu \mathrm{s}$. A comparison between $\mathrm{SSP}$ and PESTIMATION is given in 3 . 


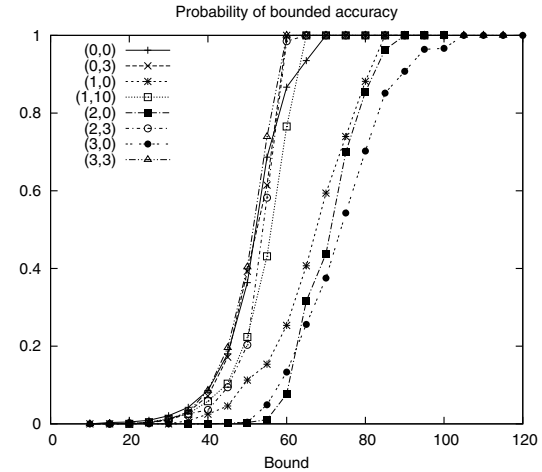

(a) Probability of satisfying bounded accuracy.

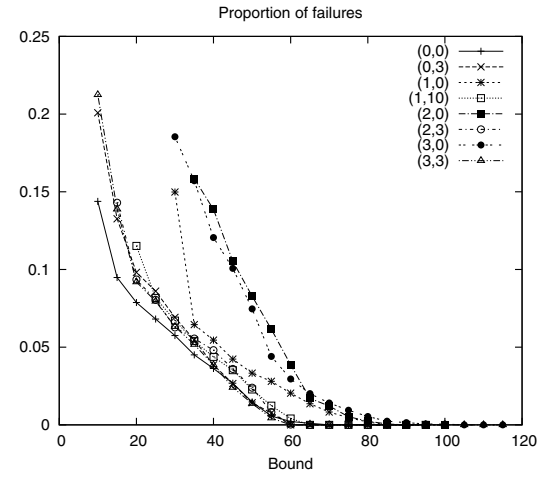

(b) Average proportion of failures.

Fig. 4. Probability of satisfying the bounded accuracy property and average proportion of failures as functions of the bound $\Delta$ for the asymmetric version of PTP

The above experiments have been conducted assuming simulations of 1000 BIP interactions and 66 rounds of the PTP protocol. Since each round of the PTP takes two minutes, this also corresponds to 132 minutes of the system's life time. We now check whether the results remain the sames if we lengthen the simulations and hence system's life time. Figure 5 shows, for Devices $(0,0)$ and $(3,0)$, the probability of synchronization for various values of $\Delta$ and various length of simulations (1000, 4000, 8000 and 10000 (660 minutes of system's life time) steps). We used PESTIMATION with a precision and a confidence of 0.1 . The best bounds do not change. However, the longer the simulations are, the more the probability tends to be either 0 or 1 depending on the bound.

Property 2: Average failure. In the previous experiment, we have computed the best bound to guarantee the bounded accuracy property. It might be the case that the bound is too high regarding the user's requirements. In such case, using the above results, we can already report on the probability for synchronization to occur properly for smaller values of the bound. We now give a finer answer by quantifying the average and worst number of failures in synchronization that occur per simulation when working with smaller bounds. For a given simulation, the proportion of failures is obtained by dividing the number of failures by the number of rounds of PTP. We will now estimate, for a simulation of 1000 steps (66 rounds of the PTP), the average and worst value for this proportion. To this purpose, we have measured (for each device) this proportion on 1199 simulations with a synchronization bound of $\Delta=50 \mu \mathrm{s}$. As an example, we obtain average proportions of 0.036 and 0.014 for Device $(0,0)$ using the symmetric and asymmetric versions of PTP respectively. As a comparison, we obtain average proportions of 0.964 and 0.075 for Device $(3,0)$. The average proportion of failures with the bound $\Delta=50 \mu s$ and the asymmetric version of PTP is given in Figure $3 \mathrm{~b}$. Figure $6 \mathrm{a}$ presents, for the sampled devices, the worst proportion of 

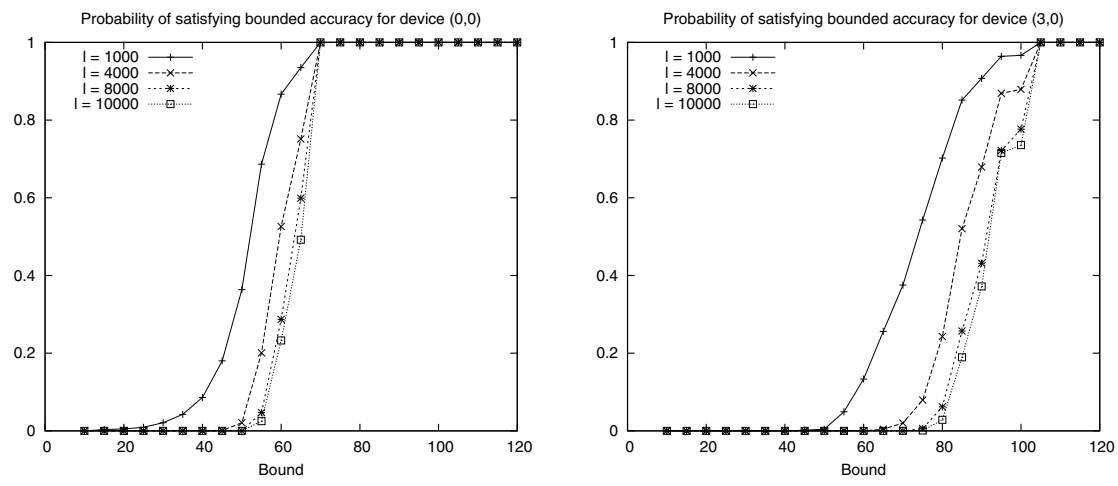

Fig. 5. Evolution of the probability of satisfying the bounded accuracy property with the length of the simulations for the asymmetric version of PTP

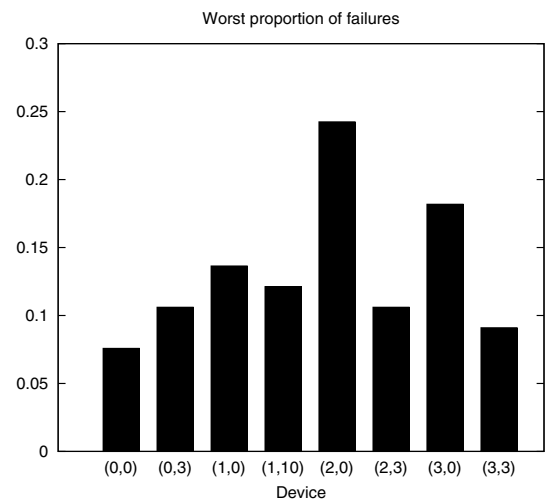

(a) Worst proportion of failures for bound $\Delta=50 \mu \mathrm{s}$.

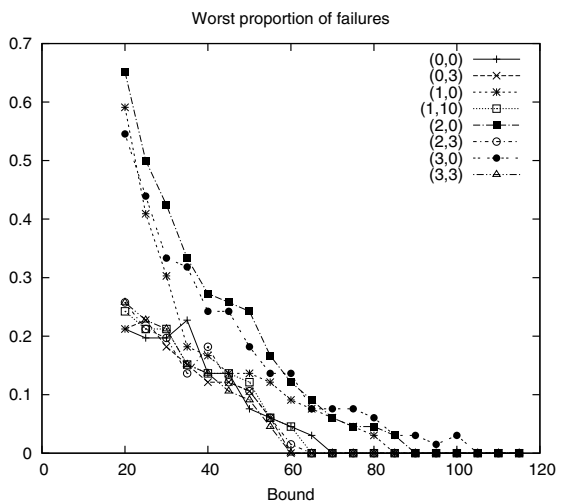

(b) Worst proportion of failures as a function of the bound $\Delta$.

Fig. 6. Worst proportion of failures for the industrial bound $\Delta=50 \mu s$ and as a function of the bound $\Delta$ for the asymmetric version of PTP

failures using the asymmetric version of PTP. The worst value is 0.25 , which is obtained for Device $(2,0)$. On the other hand, the worst value is only 0.076 for Device $(0,0)$. The experiment, which takes about 6 seconds per device, was then generalized to other values of the bound. Figures $4 \mathrm{~b}$ and $6 \mathrm{~b}$ give the average and worst proportion of failure as a function of the bound.

The above experiment gives, for several value of $\Delta$ and each device, the worst failure proportion with respect to 1199 simulations. We have also used PESTIMATION with confidence of 0.1 and precision of 0.1 to verify that this value remains the same whatever the number of simulations is. The result was then validated using SSP with precision of $10^{-3}$ and confidence of $10^{-10}$. Each experiment took approximately two minutes. Finally, we have conducted experiments to check whether the results remain for longer simulations. Figure 7 a shows that 


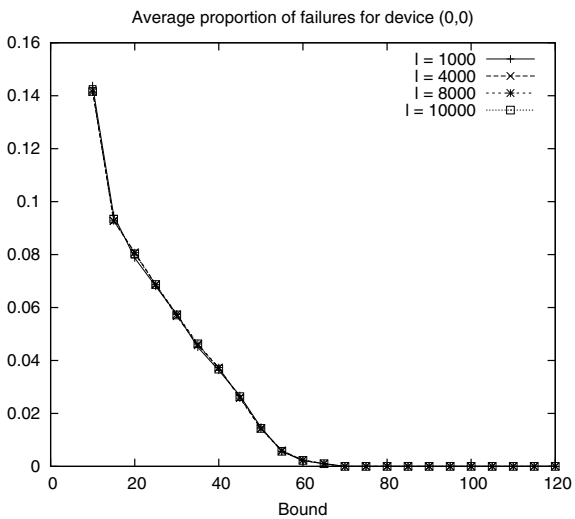

(a) Average proportion of failure for Device $(0,0)$.

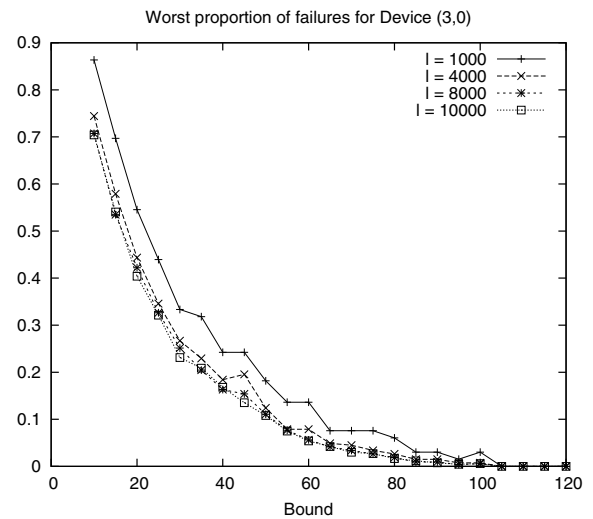

(b) Worst proportion of failure for Device $(3,0)$.

Fig. 7. Evolution of the average and worst proportion of failures with the length of the simulations for the asymmetric version of PTP

the average proportion does not change and Figure $7 \mathrm{~b}$ shows that the worst proportion decreases when the length of the simulation increases.

Clock Drift. We have considered a modified version of the stochastic PTP model with drifting clocks. Drift is used to model the fact that, due to the influence of the hardware, clocks of the master and the device may not progress as the same rate. In our model, drift is incorporated as follows: each time the clock of the server is increased by 1 time unit, the clock of the device is increased by $1+\varepsilon$ time units, with $\varepsilon \in\left[-10^{-3}, 10^{-3}\right]$. Using this modified model, we have re-done the experiments of the previous sections and observed that the result remains almost the same. This is not surprising as the value of the drift is significantly smaller than the communication jitter, and therefore it has less influence on the synchronization. A drift of 1 time unit has a much higher impact on the probability. As an example, for Device $(0,0)$, it goes from a probability of 0.387 to a probability of 0.007 . It is worth mentioning that exhaustive verification of a model with drifting clocks is not an easy task as it requires to deal with complex differential equations. When reasoning on one execution at a time, this problem is avoided.

\section{References}

1. IEEE - IEC 61588: Precision clock synchronization protocol for networked measurement and control systems (2004)

2. Basu, A., Bozga, M., Sifakis, J.: Modeling Heterogeneous Real-time Systems in BIP. In: SEFM 2006, Pune, India, September 2006, pp. 3-12 (2006) 
3. Basu, A., Bensalem, S., Bozga, M., Caillaud, B., Delahaye, B., Legay, A.: Statistical abstraction and model-checking of large heterogeneous systems. Research Report RR-7238, INRIA (March 2010), http://hal.inria.fr/inria-00466158/PDF/RR-7238.pdf

4. The BIP Toolset, http://www-verimag.imag.fr/ async/bip.php

5. Clarke, E.M., Donzé, A., Legay, A.: Statistical model checking of mixed-analog circuits with an application to a third order delta-sigma modulator. In: HVC 2008. LNCS, vol. 5394, pp. 149-163. Springer, Heidelberg (2008) (to appear)

6. Clarke, E.M., Faeder, J.R., Langmead, C.J., Harris, L.A., Jha, S.K., Legay, A.: Statistical model checking in biolab: Applications to the automated analysis of t-cell receptor signaling pathway. In: Heiner, M., Uhrmacher, A.M. (eds.) CMSB 2008. LNCS (LNBI), vol. 5307, pp. 231-250. Springer, Heidelberg (2008)

7. Grosu, R., Smolka, S.A.: Monte carlo model checking. In: Halbwachs, N., Zuck, L.D. (eds.) TACAS 2005. LNCS, vol. 3440, pp. 271-286. Springer, Heidelberg (2005)

8. Hérault, T., Lassaigne, R., Magniette, F., Peyronnet, S.: Approximate probabilistic model checking. In: Steffen, B., Levi, G. (eds.) VMCAI 2004. LNCS, vol. 2937, pp. 73-84. Springer, Heidelberg (2004)

9. Hoeffding, W.: Probability inequalities. Journal of the American Statistical Association 58, 13-30 (1963)

10. Jansen, D.N., Katoen, J.P., Oldenkamp, M., Stoelinga, M., Zapreev, I.S.: How fast and fat is your probabilistic model checker? an experimental performance comparison. In: Yorav, K. (ed.) HVC 2007. LNCS, vol. 4899, pp. 69-85. Springer, Heidelberg (2008)

11. Jha, S.K., Clarke, E.M., Langmead, C.J., Legay, A., Platzer, A., Zuliani, P.: A bayesian approach to model checking biological systems. In: Degano, P., Gorrieri, R. (eds.) CMBS 2009. LNCS, vol. 5688, pp. 218-234. Springer, Heidelberg (2009)

12. Laplante, S., Lassaigne, R., Magniez, F., Peyronnet, S., de Rougemont, M.: Probabilistic abstraction for model checking: An approach based on property testing. ACM Trans. Comput. Log. 8(4) (2007)

13. Sen, K., Viswanathan, M., Agha, G.: Statistical model checking of black-box probabilistic systems. In: Alur, R., Peled, D.A. (eds.) CAV 2004. LNCS, vol. 3114, pp. 202-215. Springer, Heidelberg (2004)

14. Wald, A.: Sequential tests of statistical hypotheses. Annals of Mathematical Statistics 16(2), 117-186 (1945)

15. Younes, H.L.S.: Verification and Planning for Stochastic Processes with Asynchronous Events. Ph.D. thesis, Carnegie Mellon (2005)

16. Younes, H.L.S., Kwiatkowska, M.Z., Norman, G., Parker, D.: Numerical vs. statistical probabilistic model checking. STTT 8(3), 216-228 (2006) 